Muss aber Politikverdrossenheit wirklich mit dem Attribut „knuffig“ bezeichnet werden (S. 212)? Was bedeutet es, wenn Misstrauen „dickflüssig“ ist (S. 14)? An vielen Stellen stößt sich der Leser an Formulierungen. Kurzum: Ein etwas nüchterner, dezenterer, weniger umgangssprachlicher Stil hätte gut getan.

Bei aller Kritik bleibt es jedoch das Verdienst der Autoren, dass sie mit einem interdisziplinären Zugang etliche Aspekte beleuchten, die die Urteilskraft beeinträchtigen. Sie diskutieren etwa, welchen Einfluss Gefühle, Vorurteile und Stereotype haben. Dabei erörtern sie das Problem der Urteilsfreiheit aus neurowissenschaftlicher Perspektive und zeigen auf, welche Argumente für die Willensfreiheit sprechen. Des Weiteren werden unterschiedliche ideengeschichtliche Strömungen wie etwa der Pragmatismus, die Diskursethik oder der Dezisionismus für die Lehre der politischen Urteilskraft fruchtbar gemacht. Wilfried von Bredow und Thomas Noetzel stellen zudem beispielhaft dar, wie „schreckliche Vereinfacher“, Verschwörungstheoretiker und Fundamentalisten jegliche Bemühungen um differenzierte Urteile zunichte machen. Trotz der genannten Schwächen kann das Buch im Saldo für einen Überblick über die gängigen Ansätze und Konzepte empfohlen werden.

Henrik Gast

\title{
Policy-Forschung als Schnittstellendisziplin: Fundierte Einführung, gekonnte Vertiefung
}

Blum, Sonja und Klaus Schubert: Politikfeldanalyse (Elemente der Politik), VS Verlag für Sozialwissenschaften, Wiesbaden 2009, 191 Seiten, € 16,95.

Schubert, Klaus und Nils C. Bandelow (Hrsg.): Lehrbuch der Politikfeldanalyse 2.0, 2. Auflage, Oldenbourg Verlag, München 2009, 486 Seiten, € 29,80.

Betrachtet man Politik als das vollständige Lösen gesellschaftlicher Problemlagen, wird der Beobachter nur selten umfassende Erfolge feststellen. Die Idealvorstellung vom politischadministrativen System als rationale Problemlösungsinstanz trägt stets auch einen normativen Überschuss in sich. Politische Prozesse gleichen vielmehr Ereignisschleifen, in denen Versuche der Problemlösung und zeitlich sowie sektoral begrenzte Teilerfolge aufeinanderfolgen. Ständig produziert das politisch-administrative System neue Vorschläge, bestehende Gesetze werden novelliert und Programme umgesetzt, die auf bestehende treffen, sie fortschreiben, korrigieren oder ergänzen. Insofern ist Politik eine gewisse Unendlichkeit eingeschrieben. Politische Prozesse in komplexen Teilsektoren funktional differenzierter Gesellschaften folgen je eigenen Rationalitäten. Die Entstehung mehr oder weniger trennscharfer Bindestrich-Politologien bildet den Treibsatz für die Begründung der Politikfeldanalyse. Bestimmend für diese mittlerweile fest etablierte Subdisziplin ist die Suche nach politikfeldspezifischen Akteurskonstellationen, Verfahrensabläufen und Ergebnissen.

Mit ihrem Einführungsband, der in der Reihe „Elemente der Politik“ erschienen ist, präsentieren Sonja Blum und Klaus Schubert eine handliche und sehr brauchbare Annäherung an die Politikfeldanalyse. Untergliedert in sieben Kapitel wird der Leser mit sämtlichen zentralen Begriffen, Konzepten und Ansätzen vertraut gemacht, die ihm Orientierung bei der Frage nach "what governments do, why they do it and what difference it makes“ (Thomas R. Dye) geben. 
Mit den schulbildenden Arbeiten von Theodore Lowi, David Easton und Hugh Heclo werden zunächst die Anfänge der Policy-Forschung innerhalb der US-amerikanischen Politikwissenschaft vorgestellt. Nach methodischen und theoretischen Ansätzen widmet sich ein Kapitel den Akteuren, Institutionen und Instrumenten aus politikfeldrelevanter Perspektive. Trotz ihrer Eigenständigkeit im Kanon politikwissenschaftlicher Disziplinen hat es die Politikfeldanalyse bis heute nicht vermocht, ein einheitliches Theorie- und Methodenrepertoire auszubilden. Dies führt dazu, dass sie sich aus dem reichhaltigen Angebot bereits erprobter Methoden und Theorien bedient. In unterschiedlichen Kontexten trifft der Leser somit immer wieder auf die große Bandbreite von Entscheidungs- und Steuerungskonzepten, die einen Erklärungsbeitrag zu Politikergebnissen leisten. Mit der kurzen Vorstellung des „Garbage Can Model“, Policy-Netzwerken, „Eisernen Dreiecken“ und des akteurzentrierten Institutionalismus drehen Sonja Blum und Klaus Schubert das Prisma politikwissenschaftlicher Erklärungsansätze jeweils ein Stück weiter ins Licht. Ein eigenes Kapitel erläutert den Policy Cycle, „das wohl am häufigsten angewandte und einflussreichste Modell der Politikfeldanalyse“ (S. 101). Mit dieser Phasenheuristik gelingt es, das schematische Systemmodell von Politik weiterzuentwickeln und die Black Box bisher unspezifizierter politischer Prozesse zu öffnen. Deutlich wird, wie sehr der analytische Blick auf jede einzelne Phase jeweils eigene Fragen und Probleme hervorhebt. So lässt sich erörtern, warum es einigen Themen gelingt, „Relevanz für ein Regierungshandeln zu entfalten, während andere im Morast der [politischen] Ursuppe verbleiben“ (S. 108 f.), welche Faktoren eine erfolgreiche Programmumsetzung begünstigen und wer Interesse an Evaluationsergebnissen hat. Einerseits ist der Policy-Forschung ein „deskriptiver Charakter“ (S. 172) vorgeworfen worden; doch andererseits hebt gerade ihre Konzentration auf Einzelfälle und ländervergleichende Studien generalisierbare Verfahrensmuster von Politik hervor.

Ein erheblich ausgreifenderes Werk legen Klaus Schubert und Nils C. Bandelow mit ihrem Lehrbuch Politikfeldanalyse 2.0 in einer vollständig überarbeiteten und erweiterten Auflage vor. Entstanden ist eine umfangreiche Sammlung von insgesamt achtzehn Beiträgen, die den Facettenreichtum der Politikfeldanalyse beleuchtet. Gegliedert nach thematischen Kategorien reichen die Analysen von den Grundlagen der Policy-Forschung, über Akteure und Institutionen bis hin zu methodischen Aspekten. Dass die Struktur des Buches auch durch die Erfahrungen der Herausgeber als Hochschullehrer inspiriert ist, lässt der didaktische Rahmen vermuten. Jedes Einzelkapitel wird neben der obligatorischen Literaturliste zudem mit einer Auswahl an Verständnis-, Transfer- sowie Problematisierungsfragen abgeschlossen.

Werner Jann und Kai Wegrich stellen mit dem Policy Cycle eine der Zentralkategorien der Politikfeldanalyse vor. Das klassische „Policy-Making“ verstehen sie „als sequentiellen Prozess der Formulierung und Umsetzung von Politikinhalten“ (S. 76). Lange Zeit war die deutsche Politikwissenschaft durch die so genannte "Gesetzgeberperspektive“ dominiert, nach deren Vorstellung Gesetze und Rechtsverordnungen zu einer unmittelbaren Problemlösung führten. Erst die genauere und isolierte Betrachtung der Implementationsphase hat Vollzugsdefizite und Konfliktniveaus einzelner Politikfelder deutlich werden lassen. Die Berücksichtigung praktischer Politikumsetzung werten beide Autoren demnach ,als eine der wichtigsten Innovationen der Policy-Forschung in den 1970er Jahren“ (S. 95). Dieses Kapitel (als Teil des Abschnitts „Basiskategorien“) lässt sich auch als Brot-und-Butter-Text der Politikfeldforschung auffassen. Hier wird sehr rasch klar, welche Mechanismen politische Prozesse beeinflussen, welche Netzwerke, Expertenarenen sowie Aushandlungsarrange- 
ments entscheidend sind und in welchen analytischen Abstufungen die Policy-Analyse den unintendierten Nebenfolgen von Politik nachspürt.

Politische Prozesse reflektieren Veränderungen des Status quo. Einerseits durch drängende Problemlagen ausgelöst, sind Statusveränderungen andererseits auch manifester Ausdruck politischen Lernens. In seinem Beitrag zeichnet Nils C. Bandelow die Konturen unterschiedlichen Lernverhaltens nach. Wertbezogene Paradigmenwechsel werden zumeist durch die Verschiebung bislang dominanter Wahrnehmungsmuster ausgelöst. Wenn bestehende Paradigmen ihre Legitimation verlieren, kann „Veränderungslernen“ (S. 319) zu neuen Interpretationen der Wirklichkeit führen. Der Advocacy-Koalitionenansatz - als Alternative zum Policy Cycle konzipiert - geht davon aus, „dass sich im Streit um bestimmte politische Probleme [...] langfristig Akteure mit übereinstimmenden allgemeinen Wertvorstellungen, Kausalannahmen und Problemwahrnehmungen zusammenschließen“ (S. 331). Andere Formen des politischen Lernens finden in den Arrangements von Politiknetzwerken statt, während das Konzept des Policy-Transfers eine zusätzliche „Erklärung für konvergente Politikentwicklung" (S. 337) anbietet.

In einem sehr lesenswerten Beitrag nimmt Göttrik Wewer das Geschäft der Politikberatung in den Blick. Wewer, vertraut mit der doppelten Perspektive des praktischen Kenners politischer Apparate und des Wissenschaftlers, beginnt seine Ausführungen mit der treffenden Beobachtung, dass es „wohl kaum eine andere Berufsgruppe [gibt], die ständig so viele gute Ratschläge bekommt wie die Politiker“ (S. 401). Beratungsleistungen kommen in vielen unterschiedlichen Gewändern daher; Gutachten, Papiere und Empfehlungen entstammen Forschungseinrichtungen, Ministerien oder Denkfabriken, doch der überwiegende Rat, den Politiker empfangen, ist „unwissenschaftlich“ (S. 413). Politische Entscheidungsträger sind bisweilen geradezu eingeschneit mit wohlmeinendem Fachwissen, um politische Ergebnisse zu verbessern. Nicht jedes Konzept ist hilfreich, nicht jeder Rat ist fundiert, „nur weil ihn ein Professor erteilt“ (S. 416). Ein Gutachten kann stets ein passendes Gegengutachten evozieren, und insofern ähnelt das Geschäft der Politikberatung einem Prozess des fortwährenden Auf- und Nachrüstens.

Gerade durch ihre Unterschiedlichkeit ergänzen sich beide Werke in vielerlei Hinsicht. Während die konzise Einführung von Sonja Blum und Klaus Schubert fundiert einen ersten Weg durch das Terrain der Politikfeldanalyse weist, vertieft und verdichtet das Lehrbuch von Klaus Schubert und Nils C. Bandelow eine Vielzahl weiterreichender Aspekte. Hieran wird rasch ersichtlich, wie sehr die Policy-Forschung zu einer unverzichtbaren Schnittstellendisziplin geworden ist. Antworten zu etlichen Forschungsfragen - von den Bedingungen erfolgreicher Reformen, über Blockadepotentiale einzelner Akteure bis hin zu Machtfragen - lassen sich daher sehr angemessen mit dem Instrumentarium der entwickelten Politikfeldanalyse finden. 